\title{
New models show gut-brain transmission of Parkinson disease pathology
}

spread of
a-synuclein
pathology
from the gut
to the brain
relied on an
intact vagus
nerve and the
presence of
endogenous
a-synuclein

The idea that $a$-synuclein pathology

in Parkinson disease (PD)

originates in the gastrointestinal

tract and is transmitted to the brain

via the vagus nerve came from

post-mortem studies published

by Heiko Braak and colleagues

in the early 2000s. However, this phenomenon has proved difficult to study in living patients, and animal models have failed to fully recapitulate this aspect of $\mathrm{PD}$ pathogenesis. Now, two groups have generated new rodent models of PD that are believed to provide the most accurate representation of gut-brain a-synuclein transmission to date.

In the first study, led by

Ted Dawson and Han Seok Ko at

Johns Hopkins University School of Medicine, Baltimore, USA, a-synuclein preformed fibrils (PFFs) were injected into the muscularis layer of the duodenum and pylorus in mice. Aggregates of pathological a-synuclein were detectable in the dorsal motor nucleus of the vagus (DMV) and the locus coeruleus 1 month after injection and subsequently spread to the amygdala,

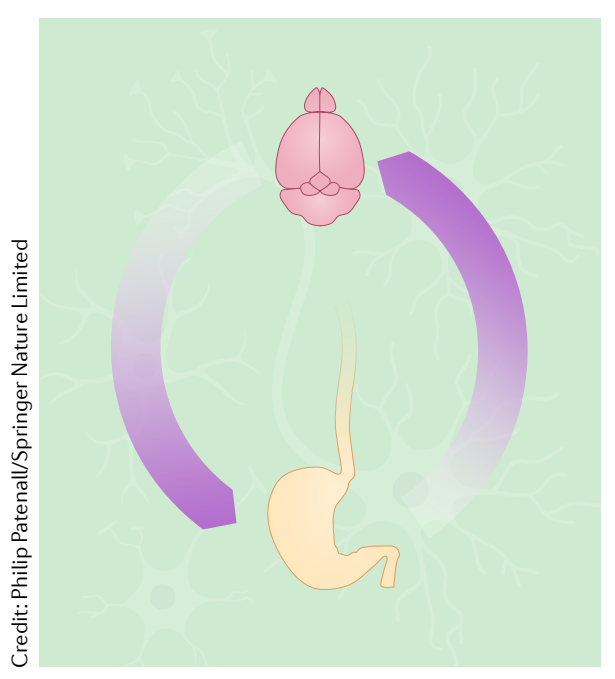

dorsal raphe nucleus and substantia nigra. The spread of pathology was paralleled by loss of dopaminergic neurons and was also associated with the emergence of motor and non-motor symptoms resembling idiopathic PD.

The researchers found that the spread of $\alpha$-synuclein pathology from the gut to the brain relied on an intact vagus nerve and the presence of endogenous $\alpha$-synuclein. "The pathological $\alpha$-synuclein did not ascend into the brain when animals underwent truncal vagotomy or endogenous $\alpha$-synuclein was depleted," comments Ko.

"We now plan to explore what parts of the vagus nerve allow misfolded a-synuclein to ascend to the brain and to investigate the underlying mechanisms."

The second study was conducted by Nathalie Van Den Berge and colleagues at Aarhus University in Denmark. The team injected human a-synuclein PFFs into the wall of the duodenum in transgenic rats that overexpressed a human form of $\alpha$-synuclein. "We anticipated that the excess gene dosage in these rats would enable the conversion of endogenous $\alpha$-synuclein to pathological aggregates, thereby facilitating the propagation of gut-induced PD pathology," explains Van Den Berge.

The investigators found evidence of transmission of $\alpha$-synuclein pathology through both sympathetic and parasympathetic pathways to the DMV and locus coeruleus. Moreover, they showed that the pathology could propagate in the opposite direction from the DMV to the stomach and could reach the heart via the cardiac sympathetic nervous system.

"Although both retrograde and anterograde vagal transport and propagation of $\alpha$-synuclein have been shown before, previous studies did not attempt to investigate bidirectional propagation in the same study protocol," says Van Den Berge. "We also found robust $\alpha$-synuclein propagation through both arms of the autonomic nervous system and demonstrated involvement of the cardiac sympathetic nervous system; these findings suggest that the pathology quickly finds its way to the heart via the autonomic connectome."

As both teams point out, these new rodent models provide valuable tools to further explore the role of the gut-brain axis in the initiation and propagation of $\mathrm{PD}$ pathology. "Our model will serve to accelerate the study of specific cellular and molecular pathways associated with the centripetal trafficking of a-synuclein from the gut to the brain in PD and related a-synucleinopathies," concludes Dawson. "It can also be used to test potential therapeutic interventions and should spur additional research that might one day allow us to prevent PD by interfering with the generation of pathological a-synuclein in the gut."

Heather Wood

ORIGINAL ARTICLES Kim, S. et al. Transneuronal propagation of pathologic $\alpha$-synuclein from the gut to the brain models Parkinson's disease. Neuron https://doi.org/10.1016/j.neuron. 2019.05 .035 (2019) | Van Den Berge, N. et al. Evidence for bidirectional and trans-synaptic parasympathetic and sympathetic propagation of alpha-synuclein in rats. Acta Neuropathol. https:// doi.org/10.1007/s00401-019-02040-w (2019) FURTHER READING Braak, H. et al. Staging of brain pathology related to sporadic Parkinson's disease. Neurobiol. Aging 24, 197-211 (2003)| Braak, H. et al. Idiopathic Parkinson's disease: possible routes by which vulnerable neuronal types may be subject to neuroinvasion by an unknown pathogen. J. Neural Transm. 110, 517-536 (2003) 